IJMMS 32:1 (2002) 47-55

PII. S0161171202006877

http://ijmms.hindawi.com

(c) Hindawi Publishing Corp.

\title{
ON A SUM ANALOGOUS TO DEDEKIND SUM AND ITS MEAN SQUARE VALUE FORMULA
}

\author{
ZHANG WENPENG
}

Received 1 March 2001

The main purpose of this paper is using the mean value theorem of Dirichlet $L$-functions to study the asymptotic property of a sum analogous to Dedekind sum, and give an interesting mean square value formula.

2000 Mathematics Subject Classification: 11N37, 11M20.

1. Introduction. For a positive integer $k$ and an arbitrary integer $h$, the classical Dedekind sum $S(h, k)$ is defined by

$$
S(h, k)=\sum_{a=1}^{k}\left(\left(\frac{a}{k}\right)\right)\left(\left(\frac{a h}{k}\right)\right)
$$

where

$$
((x))= \begin{cases}x-[x]-\frac{1}{2}, & \text { if } x \text { is not an integer; } \\ 0, & \text { if } x \text { is an integer. }\end{cases}
$$

The various properties of $S(h, k)$ were investigated by many authors. For example, Carlitz [1] obtained a reciprocity theorem of $S(h, k)$. Conrey et al. [2] studied the mean value distribution of $S(h, k)$, and first proved the following important asymptotic formula:

$$
\sum_{h=1}^{k}|S(h, k)|^{2 m}=f_{m}(k)\left(\frac{k}{12}\right)^{2 m}+O\left(\left(k^{9 / 5}+k^{2 m-1+1 /(m+1)}\right) \log ^{3} k\right),
$$

where $\sum_{h}^{\prime}$ denotes the summation over all $h$ such that $(k, h)=1$, and

$$
\sum_{n=1}^{\infty} \frac{f_{m}(n)}{n^{s}}=2 \frac{\zeta^{2}(2 m)}{\zeta(4 m)} \cdot \frac{\zeta(s+4 m-1)}{\zeta^{2}(s+2 m)} \zeta(s) .
$$

The author [4] improved the error term of (1.3) for $m=1$. In October, 2000, Todd Cochrane (personal communication) introduced a sum analogous to Dedekind sum as follows:

$$
C(h, k)=\sum_{a=1}^{k}\left(\left(\frac{\bar{a}}{k}\right)\right)\left(\left(\frac{a h}{k}\right)\right)
$$


where $\bar{a}$ defined by equation $a \bar{a} \equiv 1 \bmod k$. Then he suggested to study the arithmetical properties and mean value distribution properties of $C(h, k)$. About first problem, we have not made any progress at present. But for the second problem, we use the estimates for character sums and the mean value theorem of Dirichlet $L$-functions to prove the following main conclusion.

THEOREM 1.1. Let $k$ be any integer with $k>2$. Then we have the asymptotic formula

$$
\sum_{h=1}^{k} C^{2}(h, k)=\frac{5}{144} \phi^{2}(k) \prod_{p^{\alpha} \| k} \frac{(p+1)^{2} /\left(p^{2}+1\right)+1 / p^{3 \alpha}}{1+1 / p+1 / p^{2}}+O\left(k \exp \left(\frac{4 \ln k}{\ln \ln k}\right)\right),
$$

where $\exp (y)=e^{y}, \phi(k)$ is Euler function, $\prod_{p^{\alpha} \| k}$ denotes the product over all prime divisors of $k$ with $p^{\alpha} \mid k$ and $p^{\alpha+1} \nmid k$.

It seems that our methods are useless for mean value $\sum_{h=1}^{k} C^{2 m}(h, k)$ with $m>1$.

2. Some lemmas. To complete the proof of Theorem 1.1, we need the following lemmas.

LEMMA 2.1. Let integer $k \geq 3$ and $(h, k)=1$. Then

$$
S(h, k)=\frac{1}{\pi^{2} k} \sum_{d \mid k} \frac{d^{2}}{\phi(d)} \sum_{\substack{x \bmod d \\ x(-1)=-1}} x(h)|L(1, \chi)|^{2},
$$

where $\chi$ denotes a Dirichlet character modulo $d$ with $\chi(-1)=-1$, and $L(s, \chi)$ denotes the Dirichlet L-function corresponding to $\chi$.

Proof. See [3].

LEMMA 2.2. Let $k$ be any integer with $k>2$. Then we have the identity

$$
\sum_{h=1}^{k} C^{2}(h, k)=\sum_{d \mid k} \mu(d) \sum_{a=1}^{k} S\left(a, \frac{k}{d}\right) S(a, k)
$$

where $\mu(d)$ is Möbius function.

Proof. From the definition of $S(h, k)$ and $C(h, k)$, we have

$$
\begin{aligned}
\sum_{h=1}^{k} C^{2}(h, k) & =\sum_{h=1}^{k}\left(\sum_{a=1}^{k}\left(\left(\frac{\bar{a}}{k}\right)\right)\left(\left(\frac{a h}{k}\right)\right)\right)^{2} \\
& =\sum_{a=1}^{k} \sum_{b=1}^{k}\left(\left(\frac{\bar{a}}{k}\right)\right)\left(\left(\frac{\bar{b}}{k}\right)\right) \sum_{h=1}^{k}\left(\left(\frac{a h}{k}\right)\right)\left(\left(\frac{b h}{k}\right)\right) .
\end{aligned}
$$


Since $(a b, k)=1$, so if $h$ round through a complete residue system modulo $k$, then $\bar{b} h$ also round through a complete residue system modulo $k$. Therefore, note that the identities

$$
\sum_{b=1}^{k}=\sum_{d \mid k} \mu(d) \sum_{b=1}^{k / d}, \quad S(a, k)=S(\bar{a}, k),
$$

we have

$$
\begin{aligned}
\sum_{h=1}^{k} C^{2}(h, k) & =\sum_{a=1}^{k} \sum_{b=1}^{k}\left(\left(\frac{\bar{a}}{k}\right)\right)\left(\left(\frac{\bar{b}}{k}\right)\right) \sum_{h=1}^{k}\left(\left(\frac{a \bar{b} h}{k}\right)\right)\left(\left(\frac{h}{k}\right)\right) \\
& =\sum_{a=1}^{k} \sum_{b=1}^{k}\left(\left(\frac{\bar{a}}{k}\right)\right)\left(\left(\frac{\bar{b}}{k}\right)\right) S(a \bar{b}, k) \\
& =\sum_{a=1}^{k} \sum_{b=1}^{k}\left(\left(\frac{\bar{a}}{k}\right)\right)\left(\left(\frac{b}{k}\right)\right) S(a b, k) \\
& =\sum_{d \mid k} \mu(d) \sum_{a=1}^{k} \sum_{b=1}^{k / d}\left(\left(\frac{a b}{k / d}\right)\right)\left(\left(\frac{b}{k / d}\right)\right) S(\bar{a}, k) \\
& =\sum_{d \mid k} \mu(d) \sum_{a=1}^{k} S\left(a, \frac{k}{d}\right) S(\bar{a}, k) \\
& =\sum_{d \mid k} \mu(d) \sum_{a=1}^{k} S\left(a, \frac{k}{d}\right) S(a, k) .
\end{aligned}
$$

This proves Lemma 2.2.

LEMMA 2.3. Let $u$ and $v$ be integers with $(u, v)=d \geq 2, \chi_{u}^{0}$ the principal character $\bmod u$, and $\chi_{v}^{0}$ the principal character $\bmod v$. Then we have the asymptotic formula

$$
\begin{aligned}
& \sum_{\substack{x \bmod d \\
x(-1)=-1}}\left|L\left(1, \chi \chi_{u}^{0}\right)\right|^{2}\left|L\left(1, \chi \chi_{v}^{0}\right)\right|^{2} \\
& \quad=\frac{5 \pi^{4}}{144} \phi(d) \frac{\prod_{p \mid u v}\left(p^{2}-1\right)^{2} / p^{2}\left(p^{2}+1\right)}{\prod_{p \mid d} p^{2} /\left(p^{2}-1\right)}+O\left(\frac{\phi(d)}{d} \exp \left(\frac{3 \ln m}{\ln \ln m}\right)\right),
\end{aligned}
$$

where $\prod_{p \mid n}$ denotes the product over all prime divisors of $n,(u, v)$ denotes the greatest common divisor of $u$ and $v$, and $m=\max (u, v)$.

PRoof. Let $r(n)=\sum_{t \mid n} \chi_{u}^{0}(t) \chi_{v}^{0}(n / t), \chi$ an odd character mod $d$. Then for parameter $N \geq d$, applying Abel's identity we have

$$
\begin{aligned}
L\left(1, \chi \chi_{u}^{0}\right) L\left(1, \chi \chi_{v}^{0}\right) & =\sum_{n=1}^{\infty} \frac{\chi(n) r(n)}{n} \\
& =\sum_{1 \leq n \leq N} \frac{\chi(n) r(n)}{n}+\int_{N}^{\infty} \frac{A(y, \chi)}{y^{2}} d y,
\end{aligned}
$$


where $A(y, \chi)=\sum_{N<n \leq y} \chi(n) r(n)$. Note that the partition identities

$$
\begin{aligned}
A(y, \chi)= & \sum_{n \leq \sqrt{y}} \chi(n) \chi_{u}^{0}(n) \sum_{m \leq y / n} \chi(m) \chi_{v}^{0}(m) \\
& +\sum_{m \leq \sqrt{y}} \chi(m) \chi_{v}^{0}(m) \sum_{n \leq y / m} \chi(n) \chi_{u}^{0}(n) \\
& -\sum_{n \leq \sqrt{N}} \chi(n) \chi_{u}^{0}(n) \sum_{m \leq N / n} \chi(m) \chi_{v}^{0}(m) \\
& -\sum_{m \leq \sqrt{N}} \chi(m) \chi_{v}^{0}(m) \sum_{n \leq N / m} \chi(n) \chi_{u}^{0}(n) \\
& -\left(\sum_{n \leq \sqrt{y}} \chi(n) \chi_{u}^{0}(n)\right)\left(\sum_{n \leq \sqrt{y}} \chi(n) \chi_{v}^{0}(n)\right) \\
& +\left(\sum_{n \leq \sqrt{N}} \chi(n) \chi_{u}^{0}(n)\right)\left(\sum_{n \leq \sqrt{N}} \chi^{(n)} \chi_{v}^{0}(n)\right) .
\end{aligned}
$$

Applying Cauchy inequality and the estimates for character sums

$$
\begin{aligned}
\sum_{\chi \neq \chi_{0}}\left|\sum_{N \leq n \leq M} x(n)\right|^{2} & =\sum_{\chi \neq \chi_{0}}\left|\sum_{N \leq n \leq M \leq N+d} x(n)\right|^{2} \\
& =\phi(d) \sum_{N \leq n \leq M \leq N+d} \chi_{0}(n)-\left|\sum_{N \leq n \leq M \leq N+d} \chi_{0}(n)\right|^{2} \\
& \leq \frac{\phi^{2}(d)}{4}
\end{aligned}
$$

and note that the identities

$$
\begin{aligned}
\sum_{N \leq n \leq M} \chi(n) \chi_{u}^{0}(n) & =\sum_{d \mid u} \mu(d) \chi(d) \sum_{N / d \leq n \leq M / d} \chi(n), \\
\sum_{d \mid u}|\mu(d)| & =\prod_{p \mid u}(1+|\mu(p)|)=2^{\omega(u)},
\end{aligned}
$$

where $\omega(u)$ denotes the number of all different prime divisors of $u$. We have

$$
\begin{aligned}
\sum_{\substack{x \bmod d \\
\chi(-1)=-1}}|A(y, x)|^{2} \ll & \sqrt{y} \sum_{n \leq \sqrt{y}} \sum_{\substack{\chi \bmod d \\
\chi(-1)=-1}}\left|\sum_{m \leq y / n} \chi(m) \chi_{u}^{0}(m)\right|^{2} \\
& +\sqrt{y} \sum_{m \leq \sqrt{y}} \sum_{\substack{\chi \bmod d \\
\chi(-1)=-1}}\left|\sum_{n \leq y / m} \chi(n) \chi_{v}^{0}(n)\right|^{2} \\
& +\sum_{\substack{\chi \bmod d \\
x^{(-1)=-1}}}\left|\sum_{n \leq \sqrt{y}} \chi(n) \chi_{u}^{0}(n)\right|^{2} \times\left|\sum_{n \leq \sqrt{y}} \chi(n) \chi_{v}^{0}(n)\right|^{2} \\
\ll & \phi^{2}(d) 2^{\omega(u)+\omega(v)} .
\end{aligned}
$$


Thus from (2.11) and Cauchy inequality we get

$$
\begin{aligned}
\sum_{\substack{x \bmod d \\
x(-1)=-1}}\left|\int_{N}^{\infty} \frac{A(y, x)}{y^{2}} d y\right|^{2} & \leq \int_{N}^{\infty} \int_{N}^{\infty} \frac{1}{y^{2} z^{2}}\left(\sum_{\substack{x \bmod d \\
x(-1)=-1}}|A(y, x)| \cdot|A(z, x)|\right) d y d z \\
& \ll\left(\int_{N}^{\infty} \frac{1}{y^{2}}\left(\sum_{\substack{x \bmod d \\
x(-1)=-1}}|A(y, x)|^{2}\right)^{1 / 2} d y\right)^{2} \\
& \ll \frac{\phi^{2}(d)}{N} 2^{\omega(u)+\omega(v)} .
\end{aligned}
$$

Note that for $(a b, d)=1$, from the orthogonality relation for character sums modulo $d$ we have

$$
\sum_{\substack{x \bmod d \\ x(-1)=-1}} \chi(a) \bar{\chi}(b)= \begin{cases}\frac{1}{2} \phi(d), & \text { if } a \equiv b \bmod d \\ -\frac{1}{2} \phi(d), & \text { if } a \equiv-b \bmod d \\ 0, & \text { otherwise. }\end{cases}
$$

So that

$$
\begin{aligned}
& \sum_{\substack{x \bmod d \\
\chi(-1)=-1}}\left|\sum_{1 \leq n \leq N} \frac{\chi(n) r(n)}{n}\right|^{2} \\
& =\frac{1}{2} \phi(d) \sum_{\substack{1 \leq a, b \leq N \\
(a, b, d)=1 \\
a \equiv b(d)}} \frac{r(a) r(b)}{a b}-\frac{1}{2} \phi(d) \sum_{\substack{1 \leq a, b \leq N \\
(a b, d)=1 \\
a \equiv-b(d)}} \frac{r(a) r(b)}{a b} \\
& =\frac{1}{2} \phi(d) \sum_{\substack{1 \leq a \leq N \\
(a, d)=1}} \frac{|r(a)|^{2}}{a^{2}}+O\left(\phi(d) \sum_{b=1}^{N} \sum_{\ell=1}^{[N / d]} \frac{\tau(b) \tau(\ell d+b)}{(\ell d+b) b}\right) \\
& +O\left(\phi(d) \sum_{a=1}^{d-1} \frac{\tau(a) \tau(d-a)}{a(d-a)}\right) \\
& +O\left(\phi(d) \sum_{1 \leq a \leq N} \sum_{(1+a / d) \leq \ell \leq N / d} \frac{\tau(a) \tau(\ell d-a)}{a(\ell d-a)}\right) \\
& =\frac{1}{2} \phi(d) \sum_{\substack{n=1 \\
(n, d)=1}}^{\infty} \frac{|r(n)|^{2}}{n^{2}}+O\left(\frac{\phi(d)}{d} \exp \left(\frac{\ln N}{\ln \ln N}\right)\right) \text {, }
\end{aligned}
$$


where $\tau(n)$ is the divisor function and $r(n) \leq \tau(n) \ll \exp ((1+\epsilon) \ln 2 \ln n / \ln \ln n)$,

$$
\begin{gathered}
\sum_{\substack{x \bmod d \\
x(-1)=-1}}\left(\sum_{1 \leq n \leq N} \frac{\chi(n) r(n)}{n}\right)\left(\int_{N}^{\infty} \frac{\overline{A(y, \chi)}}{y^{2}} d y\right) \\
\ll(\ln N)^{2} \int_{N}^{\infty} \frac{1}{y^{2}}\left(\sum_{\substack{x \bmod d \\
x^{(-1)=-1}}}|A(y, \bar{x})|\right) d y \\
\ll \phi^{3 / 2}(d)(\ln N)^{2} N^{-1 / 2} 2^{\omega(u)+\omega(v)} .
\end{gathered}
$$

Taking parameter $N=d^{3}$ and note the identity

$$
\sum_{\substack{n=1 \\(n, d)=1}}^{\infty} \frac{|r(n)|^{2}}{n^{2}}=\frac{5 \pi^{4}}{72} \frac{\prod_{p \mid u v}\left(p^{2}-1\right)^{2} / p^{2}\left(p^{2}+1\right)}{\prod_{p \mid d} p^{2} /\left(p^{2}-1\right)} .
$$

From (2.11), (2.12), (2.14), and (2.15) we obtain

$$
\begin{aligned}
& \sum_{\substack{x \bmod d \\
x(-1)=-1}}\left|L\left(1, \chi x_{u}^{0}\right)\right|^{2}\left|L\left(1, \chi x_{v}^{0}\right)\right|^{2} \\
& \quad=\frac{5 \pi^{4}}{144} \phi(d) \frac{\prod_{p \mid u v}\left(p^{2}-1\right)^{2} / p^{2}\left(p^{2}+1\right)}{\prod_{p \mid d} p^{2} /\left(p^{2}-1\right)}+O\left(\frac{\phi(d)}{d} \exp \left(\frac{3 \ln m}{\ln \ln m}\right)\right) .
\end{aligned}
$$

This proves Lemma 2.3.

LEMMA 2.4. Let $p$ be a prime, and let $\alpha, \beta$ be nonnegative integers with $\beta \geq \alpha$. Then we have the identity

$$
\begin{aligned}
\sum_{d_{1} \mid p^{\beta}} \sum_{d_{2} \mid p^{\alpha}} \frac{d_{1}^{2} d_{2}^{2}}{\phi\left(d_{1}\right) \phi\left(d_{2}\right)} \phi(d) \frac{\prod_{p_{1} \mid d_{1} d_{2}}\left(p_{1}^{2}-1\right)^{2} / p_{1}^{2}\left(p_{1}^{2}+1\right)}{\prod_{p_{1} \mid d} p_{1}^{2} /\left(p_{1}^{2}-1\right)} \\
=p^{3 \alpha} \frac{(1+1 / p)^{2}-1 / p^{3 \alpha+1}}{1+1 / p+1 / p^{2}}+\frac{\left(p^{2}-1\right)^{2} p^{2 \alpha}\left(p^{\beta}-p^{\alpha}\right)}{(p-1)^{2}\left(p^{2}+1\right)},
\end{aligned}
$$

where $d=\left(d_{1}, d_{2}\right)$ denotes the greatest common divisors of $d_{1}$ and $d_{2}$.

Proof. Note that $d=\left(d_{1}, d_{2}\right)$, we have

$$
\begin{aligned}
& \sum_{d_{1} \mid p^{\alpha}} \sum_{d_{2} \mid p^{\alpha}} \frac{d_{1}^{2} d_{2}^{2}}{\phi\left(d_{1}\right) \phi\left(d_{2}\right)} \phi(d) \frac{\prod_{p_{1} \mid d_{1} d_{2}}\left(p_{1}^{2}-1\right)^{2} / p_{1}^{2}\left(p_{1}^{2}+1\right)}{\prod_{p_{1} \mid d} p_{1}^{2} /\left(p_{1}^{2}-1\right)} \\
& \quad=\sum_{u=0}^{\alpha} \sum_{v=0}^{\alpha} \frac{p^{2 u+2 v}}{\phi\left(p^{u}\right) \phi\left(p^{v}\right)} \phi\left(\left(p^{u}, p^{v}\right)\right) \frac{\prod_{p_{1} \mid p^{u+v}}\left(p_{1}^{2}-1\right)^{2} / p_{1}^{2}\left(p_{1}^{2}+1\right)}{\prod_{p_{1} \mid\left(p^{u}, p^{v}\right)} p_{1}^{2} /\left(p_{1}^{2}-1\right)}
\end{aligned}
$$




$$
\begin{aligned}
& =1+2 \sum_{\beta=1}^{\alpha} \frac{p^{2 \beta}}{\phi\left(p^{\beta}\right)} \frac{\left(p^{2}-1\right)^{2}}{p^{2}\left(p^{2}+1\right)}+\sum_{\beta=1}^{\alpha} \frac{p^{4 \beta}}{\phi\left(p^{\beta}\right)} \frac{\left(p^{2}-1\right)^{3}}{p^{4}\left(p^{2}+1\right)} \\
& +2 \sum_{\beta=1}^{\alpha-1} \sum_{\gamma=\beta+1}^{\alpha} \frac{p^{2 \beta} p^{2 \gamma}}{\phi\left(p^{\gamma}\right)} \frac{\left(p^{2}-1\right)^{3}}{p^{4}\left(p^{2}+1\right)} \\
& =1+2 \frac{\left(p^{\alpha}-1\right)\left(p^{2}-1\right)^{2}}{(p-1)^{2}\left(p^{2}+1\right)}+\frac{\left(p^{3 \alpha}-1\right)\left(p^{2}-1\right)^{3}}{\left(p^{3}-1\right)(p-1)\left(p^{2}+1\right)} \\
& +2 \sum_{\beta=1}^{\alpha-1} \frac{p^{3 \beta}\left(p^{\alpha-\beta}-1\right)}{(p-1)^{2}} \frac{\left(p^{2}-1\right)^{3}}{p^{2}\left(p^{2}+1\right)} \\
& =1+2 \frac{\left(p^{\alpha}-1\right)\left(p^{2}-1\right)^{2}}{(p-1)^{2}\left(p^{2}+1\right)}+\frac{\left(p^{3 \alpha}-1\right)\left(p^{2}-1\right)^{3}}{\left(p^{3}-1\right)(p-1)\left(p^{2}+1\right)} \\
& +2 \frac{\left(p^{3 \alpha-2}-p^{\alpha}\right)\left(p^{2}-1\right)^{2}}{(p-1)^{2}\left(p^{2}+1\right)}-2 \frac{p\left(p^{3 \alpha-3}-1\right)\left(p^{2}-1\right)^{3}}{\left(p^{3}-1\right)(p-1)^{2}\left(p^{2}+1\right)} \\
& =\frac{p^{4 \alpha}}{\phi\left(p^{\alpha}\right)}\left(1-\frac{1}{p^{3}}\right)^{-1}\left[\left(1-\frac{1}{p^{2}}\right)^{2}-\frac{1}{p^{3 \alpha+1}}\left(1-\frac{1}{p}\right)^{2}\right] \\
& =p^{3 \alpha}\left(1+\frac{1}{p}+\frac{1}{p^{2}}\right)^{-1}\left[\left(1+\frac{1}{p}\right)^{2}-\frac{1}{p^{3 \alpha+1}}\right] \text {, } \\
& \sum_{u=0}^{\alpha} \sum_{v=\alpha+1}^{\beta} \frac{p^{2 u+2 v}}{\phi\left(p^{u}\right) \phi\left(p^{v}\right)} \phi\left(\left(p^{u}, p^{v}\right)\right) \frac{\prod_{p_{1} \mid p^{u+v}}\left(p_{1}^{2}-1\right)^{2} / p_{1}^{2}\left(p_{1}^{2}+1\right)}{\prod_{p_{1} \mid\left(p^{u}, p^{v}\right)} p_{1}^{2} /\left(p_{1}^{2}-1\right)} \\
& =\sum_{j=0}^{\beta-\alpha-1} \frac{p^{j+\alpha+2}}{p-1} \frac{\left(p^{2}-1\right)^{2}}{p^{2}\left(p^{2}+1\right)}+\sum_{i=1}^{\alpha} p^{2 i} \sum_{j=0}^{\beta-\alpha-1} \frac{p^{j+\alpha+2}}{p-1} \frac{\left(p^{2}-1\right)^{3}}{p^{4}\left(p^{2}+1\right)} \\
& =\frac{p^{\alpha+2}\left(p^{\beta-\alpha}-1\right)}{(p-1)^{2}} \frac{\left(p^{2}-1\right)^{2}}{p^{2}\left(p^{2}+1\right)}+\frac{p^{2}\left(p^{2 \alpha}-1\right)}{p^{2}-1} \frac{p^{\alpha+2}\left(p^{\beta-\alpha}-1\right)}{(p-1)^{2}} \frac{\left(p^{2}-1\right)^{3}}{p^{4}\left(p^{2}+1\right)} \\
& =\frac{\left(p^{2}-1\right)^{2} p^{2 \alpha}\left(p^{\beta}-p^{\alpha}\right)}{(p-1)^{2}\left(p^{2}+1\right)} .
\end{aligned}
$$

Now combining (2.19) and (2.20) we have

$$
\begin{aligned}
\sum_{d_{1} \mid p^{\alpha}} & \sum_{d_{2} \mid p^{\beta}} \frac{d_{1}^{2} d_{2}^{2}}{\phi\left(d_{1}\right) \phi\left(d_{2}\right)} \phi\left(\left(d_{1}, d_{2}\right)\right) \frac{\prod_{p_{1} \mid d_{1} d_{2}}\left(p_{1}^{2}-1\right)^{2} / p_{1}^{2}\left(p_{1}^{2}+1\right)}{\prod_{p_{1} \mid\left(d_{1}, d_{2}\right)} p_{1}^{2} /\left(p_{1}^{2}-1\right)} \\
= & \sum_{u=0}^{\alpha} \sum_{v=0}^{\alpha} \frac{p^{2 u+2 v}}{\phi\left(p^{u}\right) \phi\left(p^{v}\right)} \phi\left(\left(p^{u}, p^{v}\right)\right) \frac{\prod_{p_{1} \mid p^{u+v}}\left(p_{1}^{2}-1\right)^{2} / p_{1}^{2}\left(p_{1}^{2}+1\right)}{\prod_{p_{1} \mid\left(p^{u}, p^{v}\right)} p_{1}^{2} /\left(p_{1}^{2}-1\right)} \\
& +\sum_{u=0}^{\alpha} \sum_{v=\alpha+1}^{\beta} \frac{p^{2 u+2 v}}{\phi\left(p^{u}\right) \phi\left(p^{v}\right)} \phi\left(\left(p^{u}, p^{v}\right)\right) \frac{\prod_{p_{1} \mid p^{u+v}}\left(p_{1}^{2}-1\right)^{2} / p_{1}^{2}\left(p_{1}^{2}+1\right)}{\prod_{p_{1} \mid\left(p^{u}, p^{v}\right)} p_{1}^{2} /\left(p_{1}^{2}-1\right)} \\
= & p^{3 \alpha} \frac{(1+1 / p)^{2}-1 / p^{3 \alpha+1}}{1+1 / p+1 / p^{2}}+\frac{\left(p^{2}-1\right)^{2} p^{2 \alpha}\left(p^{\beta}-p^{\alpha}\right)}{(p-1)^{2}\left(p^{2}+1\right)} .
\end{aligned}
$$

This proves Lemma 2.4. 
3. Proof of the theorem. In this section, we complete the proof of Theorem 1.1. Let $k$ be an integer with $k \geq 3$. Then applying Lemmas 2.1 and 2.2 we have

$$
\begin{aligned}
& \sum_{h=1}^{k} C^{2}(h, k)=\sum_{d \mid k} \mu(d) \sum_{a=1}^{k} S\left(a, \frac{k}{d}\right) S(a, k) \\
& =\sum_{d \mid k} \mu(d) \sum_{a=1}^{k}\left(\frac{d}{\pi^{2} k} \sum_{u \mid k / d} \frac{u^{2}}{\phi(u)} \sum_{\substack{x \bmod u \\
\chi(-1)=-1}} \chi(a)|L(1, \chi)|^{2}\right) \\
& \times\left(\frac{1}{\pi^{2} k} \sum_{v \mid k} \frac{v^{2}}{\phi(v)} \sum_{\substack{x \bmod v \\
\chi(-1)=-1}} \chi(a)|L(1, \chi)|^{2}\right) \\
& =\frac{1}{k^{2} \pi^{4}} \sum_{d \mid k} \mu(d) d \sum_{u \mid k / d} \sum_{v \mid k} \frac{u^{2} v^{2}}{\phi(u) \phi(v)} \\
& \times \sum_{\substack{\chi_{1}\left(\bmod u \\
\chi_{1}(-1)=-1\right.}} \sum_{\substack{\chi_{2} \bmod v \\
\chi_{2}(-1)=-1}} \sum_{a=1}^{k} \chi_{1}(a) \chi_{2}(a)\left|L\left(1, \chi_{1}\right)\right|^{2}\left|L\left(1, \chi_{2}\right)\right|^{2} .
\end{aligned}
$$

For each $\chi_{1} \bmod u$, it is clear that there exists one and only one $k_{1} \mid u$ with a unique primitive character $\chi_{k_{1}}^{1} \bmod k_{1}$ such that $\chi_{1}=\chi_{k_{1}}^{1} \chi_{u}^{0}$, here $\chi_{u}^{0}$ denotes the principal character $\bmod u$. Similarly, we also have $\chi_{2}=\chi_{k_{2}}^{2} \chi_{v}^{0}$, here $k_{2} \mid v$ and $\chi_{k_{2}}^{2}$ is a primitive character $\bmod k_{2}$. Note that $u \mid k$ and $v \mid k$, from the orthogonality of characters we have

$$
\begin{aligned}
\sum_{a=1}^{k} \chi_{1}(a) \chi_{2}(a) & =\sum_{a=1}^{k}\left[\chi_{k_{1}}^{1}(a) \chi_{k}^{0}(a)\right]\left[\chi_{k_{2}}^{2}(a) \chi_{k}^{0}(a)\right] \\
& = \begin{cases}\phi(k), & \text { if } k_{1}=k_{2}, \chi_{k_{1}}^{1}=\overline{\chi_{k_{2}}^{2}} \\
0, & \text { otherwise. }\end{cases}
\end{aligned}
$$

Let $d_{1}=(u, v)$. If $k_{1}=k_{2}$ and $\chi_{k_{1}}^{1}=\overline{\chi_{k_{2}}^{2}}$, then $\chi_{k_{1}}^{1} \chi_{d_{1}}^{0}$ is also a character $\bmod d_{1}$. So from (3.1), (3.2), and Lemma 2.3 we have

$$
\begin{aligned}
\sum_{h=1}^{k} C^{2}(h, k)= & \frac{\phi(k)}{k^{2} \pi^{4}} \sum_{d \mid k} \mu(d) d \sum_{u \mid k / d} \sum_{v \mid k} \frac{u^{2} v^{2}}{\phi(u) \phi(v)} \sum_{\substack{x \bmod (u, v) \\
X(-1)=-1}}\left|L\left(1, \chi x_{u}^{0}\right)\right|^{2}\left|L\left(1, \chi x_{v}^{0}\right)\right|^{2} \\
= & \frac{\phi(k)}{k^{2} \pi^{4}} \sum_{d \mid k} \sum_{u \mid k / d} \sum_{v \mid k} \frac{\mu(d) d u^{2} v^{2}}{\phi(u) \phi(v)}\left\{\frac{5 \pi^{4}}{144} \phi((u, v)) \frac{\prod_{p \mid u v}\left(p^{2}-1\right)^{2} / p^{2}\left(p^{2}+1\right)}{\prod_{p \mid(u, v)} p^{2} /\left(p^{2}-1\right)}\right. \\
& \left.+O\left(\exp \left(\frac{3 \ln k}{\ln \ln k}\right)\right)\right\} \\
= & \frac{5 \phi(k)}{144 k^{2}} \sum_{d \mid k} \sum_{u \mid k / d} \sum_{v \mid k} \frac{\mu(d) d u^{2} v^{2}}{\phi(u) \phi(v)} \phi((u, v)) \frac{\prod_{p \mid u v}\left(p^{2}-1\right)^{2} / p^{2}\left(p^{2}+1\right)}{\prod_{p \mid(u, v)} p^{2} /\left(p^{2}-1\right)} \\
& +O\left(k \exp \left(\frac{4 \ln k}{\ln \ln k}\right)\right) .
\end{aligned}
$$


Since $\phi(n)$ and $\mu(n)$ are multiplicative functions, so from the multiplicative properties of these functions, (3.3) and Lemma 2.4 and note that the identities (for any multiplicative functions $f(u)$ and $g(v)$ )

$$
\begin{aligned}
\sum_{d \mid k} \mu(d) d \sum_{u \mid k / d} \sum_{v \mid k} f(u) g(v) & =\prod_{p^{\alpha} \| k}\left[\sum_{u \mid p^{\alpha}} \sum_{v \mid p^{\alpha}} f(u) g(v)-p \sum_{u \mid p^{\alpha-1}} \sum_{v \mid p^{\alpha}} f(u) g(v)\right] \\
p^{3 \alpha} \frac{(1+1 / p)^{2}-1 / p^{3 \alpha+1}}{1+1 / p+1 / p^{2}}-p & {\left[p^{3 \alpha-3} \frac{(1+1 / p)^{2}-1 / p^{3 \alpha-2}}{1+1 / p+1 / p^{2}}+\frac{\left(p^{2}-1\right)^{2} p^{2 \alpha-2}\left(p-p^{\alpha-1}\right)}{(p-1)^{2}\left(p^{2}+1\right)}\right] } \\
& =\frac{p^{3 \alpha}(1-1 / p)}{1+1 / p+1 / p^{2}}\left(\frac{(p+1)^{2}}{p^{2}+1}+\frac{1}{p^{3 \alpha}}\right),
\end{aligned}
$$

we have

$$
\begin{aligned}
\sum_{h=1}^{k} C^{2}(h, k)= & \sum_{d \mid k} \mu(d) \sum_{a=1}^{k} S\left(a, \frac{k}{d}\right) S(a, k) \\
= & \frac{5}{144} \frac{\phi(k)}{k^{2}} \prod_{p^{\alpha} \| k}\left\{\sum_{d \mid p^{\alpha}} \mu(d) d \sum_{u \mid p^{\alpha} / d} \sum_{v \mid p^{\alpha}} \frac{u v}{\phi(u) \phi(v)} \phi((u, v))\right. \\
& \left.\times \frac{\prod_{p_{1} \mid u v}\left(p_{1}^{2}-1\right)^{2} / p_{1}^{2}\left(p_{1}^{2}+1\right)}{\prod_{p_{1} \mid(u, v)} p_{1}^{2} /\left(p_{1}^{2}-1\right)}\right\} \\
& +O\left(k \exp \left(\frac{4 \ln k}{\ln \ln k}\right)\right) \\
= & \frac{5}{144} \phi^{2}(k) \prod_{p^{\alpha}|| k} \frac{(p+1)^{2} /\left(p^{2}+1\right)+1 / p^{3 \alpha}}{1+1 / p+1 / p^{2}}+O\left(k \exp \left(\frac{4 \ln k}{\ln \ln k}\right)\right) .
\end{aligned}
$$

This completes the proof of Theorem 1.1.

ACKNOWLEDGMent. This work was supported by the National Natural Science Foundation of China (NSFC) and the Shaanxi Province Natural Science Foundation of China (PNSF).

\section{REFERENCES}

[1] L. Carlitz, A reciprocity theorem of Dedekind sums, Pacific J. Math. 3 (1953), 523-527.

[2] J. B. Conrey, E. Fransen, R. Klein, and C. Scott, Mean values of Dedekind sums, J. Number Theory 56 (1996), no. 2, 214-226.

[3] W. Zhang, On the mean values of Dedekind sums, J. Théor. Nombres Bordeaux 8 (1996), no. 2, 429-442.

[4] _ A note on the mean square value of the Dedekind sums, Acta Math. Hungar. 86 (2000), no. 4, 275-289.

ZHANG WeNPENG: RESEARCH CENTER FOR BASIC SCIENCE, XI'AN JIAOTONG UNIVERSITY, XI'AN, SHAANXI, CHINA 


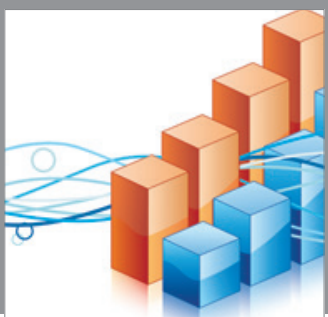

Advances in

Operations Research

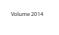

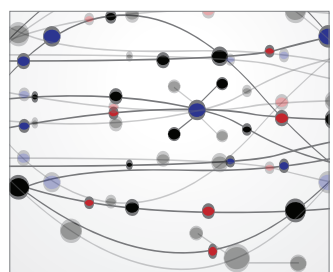

\section{The Scientific} World Journal
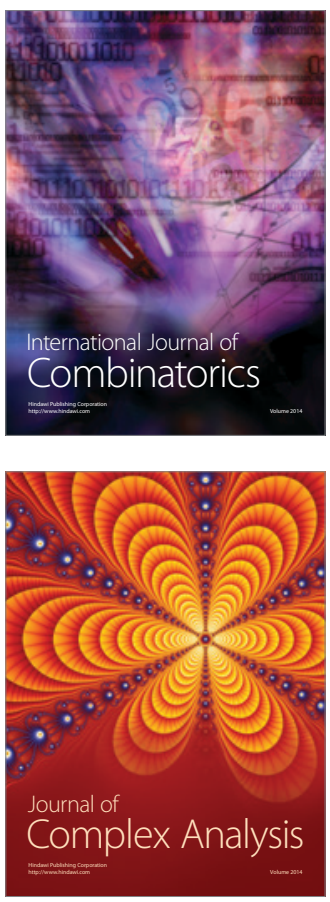

International Journal of

Mathematics and

Mathematical

Sciences
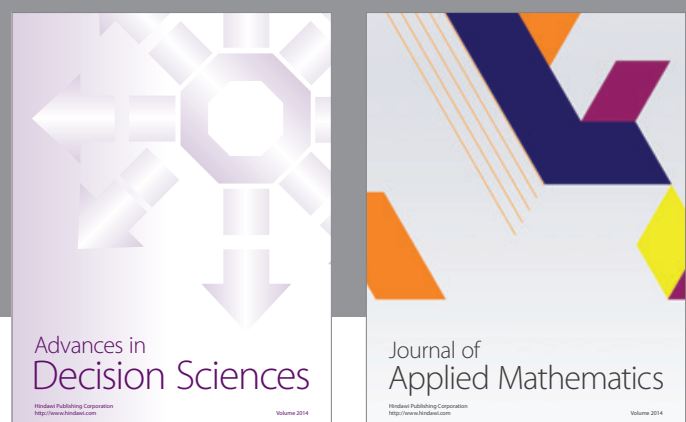

Journal of

Applied Mathematics
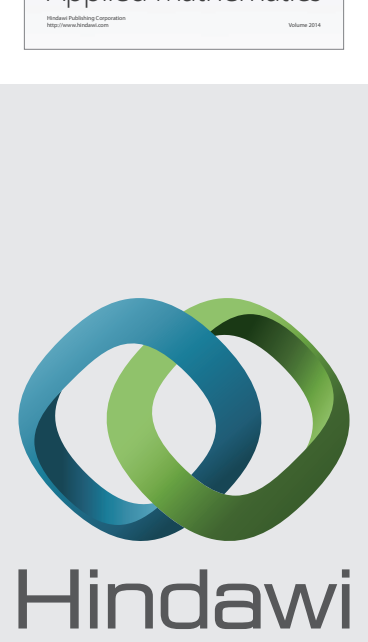

Submit your manuscripts at http://www.hindawi.com
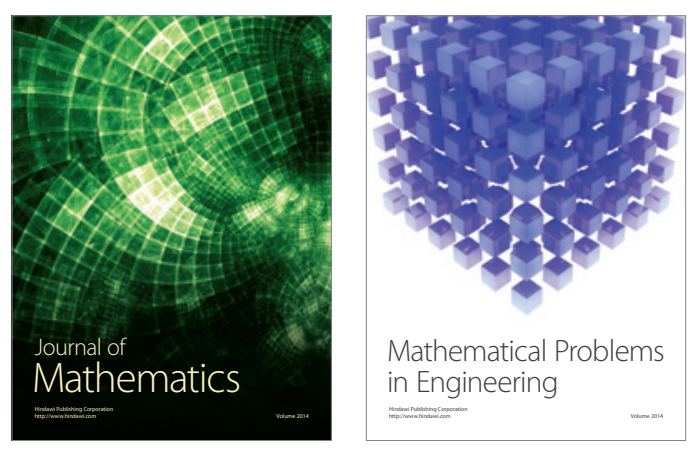

Mathematical Problems in Engineering
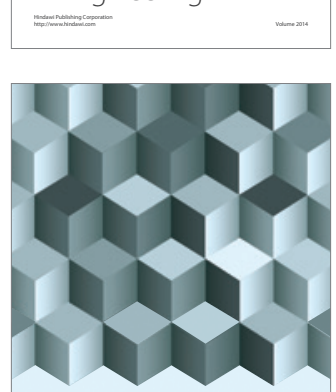

Journal of

Function Spaces
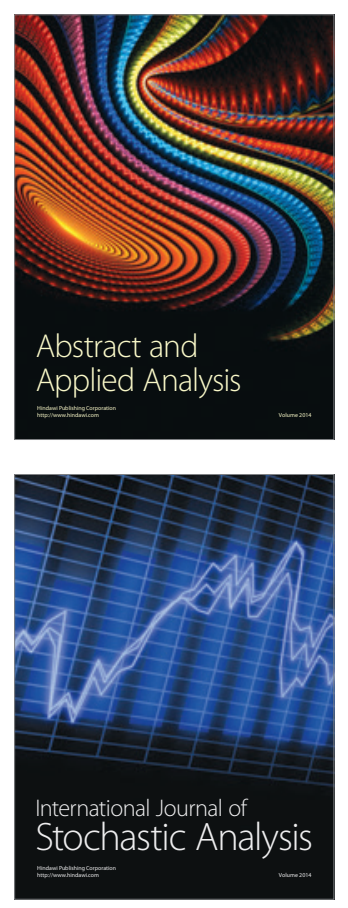

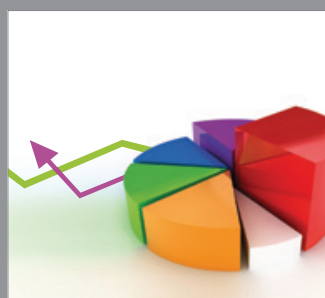

ournal of

Probability and Statistics

Promensencen
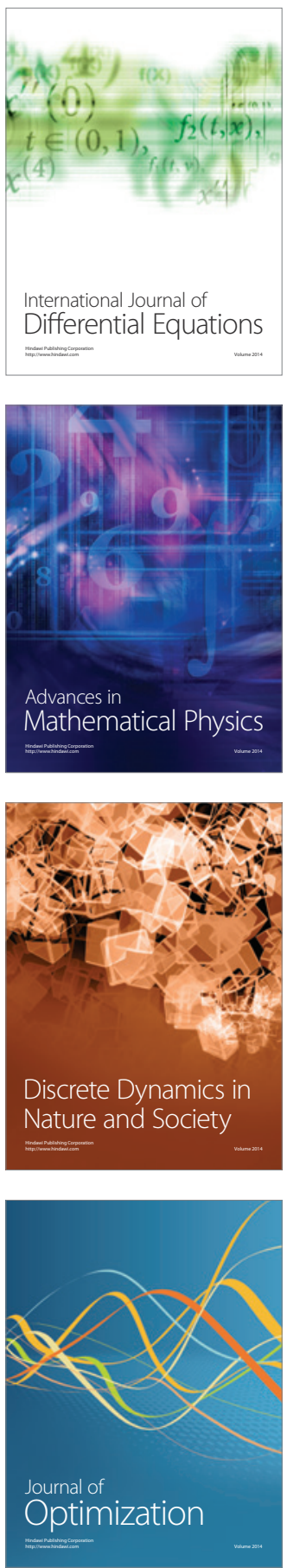